\title{
Evolutionary Patterns of MHC Class II B in Owls and Their Implications for the Understanding of Avian MHC Evolution
}

\author{
Reto Burri,* Hélène Niculita Hirzel, $\uparrow$ Nicolas Salamin, $\dagger$ Alexandre Roulin, $\dagger$ and Luca Fumagalli* \\ *Laboratory for Conservation Biology, Department of Ecology and Evolution, Biophore, University of Lausanne, Lausanne, \\ Switzerland; and †Department of Ecology and Evolution, Biophore, University of Lausanne, Lausanne, Switzerland
}

\begin{abstract}
Owing to its special mode of evolution and central role in the adaptive immune system, the major histocompatibility complex (MHC) has become the focus of diverse disciplines such as immunology, evolutionary ecology, and molecular evolution. MHC evolution has been studied extensively in diverse vertebrate lineages over the last few decades, and it has been suggested that birds differ from the established mammalian norm. Mammalian MHC genes evolve independently, and duplication history (i.e., orthology) can usually be traced back within lineages. In birds, this has been observed in only 3 pairs of closely related species. Here we report strong evidence for the persistence of orthology of MHC genes throughout an entire avian order. Phylogenetic reconstructions of MHC class II B genes in 14 species of owls trace back orthology over tens of thousands of years in exon 3. Moreover, exon 2 sequences from several species show closer relationships than sequences within species, resembling transspecies evolution typically observed in mammals. Thus, although previous studies suggested that long-term evolutionary dynamics of the avian MHC was characterized by high rates of concerted evolution, resulting in rapid masking of orthology, our results question the generality of this conclusion. The owl MHC thus opens new perspectives for a more comprehensive understanding of avian MHC evolution.
\end{abstract}

\section{Introduction}

The major histocompatibility complex (MHC) is a highly variable gene family that is crucial to the vertebrate immune system (Klein 1986). Apart from having a role in disease resistance, MHC genes are of particular interest because of their outstanding patterns of molecular evolution. These patterns have been extensively studied in mammals (Nei and Hughes 1992; Nei et al. 1997; Gu and Nei 1999). Classical MHC genes exhibit the highest levels of allelic diversity and amino acid polymorphism known in vertebrates (Geraghty et al. 2002; Bernatchez and Landry 2003). The high level of MHC polymorphism has been attributed to balancing selection, which favors high levels of allelic diversity within individuals (Doherty and Zinkernagel 1975; Hughes and Yeager 1998; Penn et al. 2002). Some mammalian MHC allelic lineages are tens of millions of years old, often predating the divergence time of the species carrying the alleles (Figueroa et al. 1988; Lawlor et al. 1988). Consequently, phylogenetic reconstructions reveal transspecies polymorphism with alleles from different species showing closer relationships than alleles from the same species (Klein et al. 1998).

Besides balancing selection that shapes levels of $\mathrm{MHC}$ diversity at the allelic level, MHC evolution is characterized by frequent events of gene duplication (birth) and gene loss (death), thereby fitting a birth-and-death model of molecular evolution (Nei and Hughes 1992; Nei et al. 1997). Although the overall structure and function of the MHC are conserved throughout vertebrates, pronounced differences in the genomic organization of the MHC do occur between vertebrate lineages (Wittzell et al. 1998; Beck and Trowsdale 2000; Kulski et al. 2002; Kelley et al. 2005), particularly between mammals and birds (Edwards et al. 1995; Hess and Edwards 2002). In most lineages, including mammals, gene

Key words: birds, gene duplication, major histocompatibility complex, molecular evolution, orthology, Strigiformes.

E-mail: reto.burri@unil.ch

Mol. Biol. Evol. 25(6):1180-1191. 2008

doi:10.1093/molbev/msn065

Advance Access publication March 20, 2008 duplicates evolve independently from each other after speciation. Thus, duplication history within lineages can usually be traced back by phylogenetic reconstructions because MHC sequences form orthologous clusters, grouping by gene rather than by species (Nei et al. 1997; Gu and Nei 1999; Nei and Rooney 2005). In contrast, avian MHC genes usually cluster by species, indicating recent duplication or elevated rates of concerted evolution, that is, frequent genetic exchange between duplicate genes by recombination or gene conversion (Edwards et al. 1995; Hess and Edwards 2002). The homogenizing effect of recombination masks orthology and impedes the reconstruction of duplication history. So far, orthology in the avian MHC has been detected only 3 times and always in pairs of closely related species, that is, in the MHC class II B (MHCIIB) of chicken (Gallus gallus) and pheasant (Phasianus colchicus) (Wittzell et al. 1999), Chatham Island robin (Petroica traversi) and South Island robin (Petroica australis) (Miller and Lambert 2004), and chicken and black grouse (Tetrao tetrix) (Strand et al. 2007).

Several authors have pointed out that birth-and-death and concerted evolution are not mutually exclusive processes but may occur over different timescales (Edwards et al. 1999; Wittzell et al. 1999). Concerted evolution might act on closely related loci over the short term and mask orthology. In contrast, birth-and-death processes lead to MHC reorganization and nonorthology of MHC genes among lineages over longer time periods. In birds, the elevated rates of concerted evolution presumably shorten the period of time during which signals of orthology are detectable. Increased understanding of avian MHC evolution thus calls for studies that include a number of rather closely related species. Previous studies considering multiple species almost exclusively used information from MHCIIB exon 2 (Vincek et al. 1997; Edwards et al. 1999; Tsuda et al. 2001; Jarvi et al. 2004). Phylogenetic reconstructions based on exon 2 are rather ambiguous as this region codes for the sites involved in antigen binding and are under strong positive selection (Hughes and Yeager 1998). Therefore, resulting phylogenetic trees may reflect selection rather than duplication history (Reusch and Langefors 2005). 
Introns and exon 3, which contains the CD4-binding site involved in T lymphocyte binding, have been shown to provide more information about the history of gene duplication (Edwards et al. 1999; Kriener et al. 2001), but the few studies that have examined these regions considered only a limited number of species (Edwards et al. 1995; Wittzell et al. 1999; Miller and Lambert 2004; Kikkawa et al. 2005).

Here we studied the evolution of avian MHC genes over successive speciation events. We combined sampling of a monophyletic group of species with sequence information from both exons 2 and 3 of MHCIIB. We reconstructed the phylogenetic relationships of MHCIIB genes among 14 species of owls (order Strigiformes; families Strigidae and Tytonidae) and provide strong evidence of avian MHC genes in which orthology has been retained over tens of millions of years. Together with transspecies evolution in exon 2 for several species, this finding reflects the patterns observed in mammals and challenges the present view of avian MHC evolution.

\section{Materials and Methods}

RNA Isolation and Reverse Transcriptase-Polymerase Chain Reaction

Total RNA was extracted by TRIzol (Invitrogen, Carlsbad, CA) from fresh blood samples of one individual of each of 10 owl species: Tengmalm's owl (Aegolius funereus), spotted eagle owl (Bubo africanus), Eurasian eagle owl (Bubo bubo), snowy owl (Bubo scandiacus), Eurasian pygmy owl (Glaucidium passerinum), European scops owl (Otus scops), tawny owl (Strix aluco), great gray owl (Strix nebulosa), Ural owl (Strix uralensis), and northern hawk owl (Surnia ulula), from spleen of short-eared owl (Asio flammeus) and barn owl (Tyto alba), and from liver of long-eared owl (Asio otus) and little owl (Athene noctua). After DNaseI treatment using the RNeasy Mini Kit (Qiagen, Hilden, Germany), first-strand cDNA was synthesized from $1 \mu \mathrm{g}$ of total RNA with $2.5 \mu \mathrm{M}$ oligo-dT (T-11-VN) and 200 U SuperScript III Reverse Transcriptase (Invitrogen).

\section{MHCIIB Amplification, Cloning, and Sequencing}

Primers Tyal-ex1F (5'-TGT GGG GGG AGT TGG GGC TGT GCT G-3') and Tyal-ex3-3'R (5'-AGG CTG ACG TGC TCC ACC TG-3') were used in polymerase chain reactions (PCRs) on $200 \mathrm{ng}$ cDNA. These primers amplify a 558-bp fragment from exon 1 to the $3^{\prime}$ end of exon 3 in barn owl MHCIIB genes that are expressed in spleen, liver, and blood in this species (Burri R, Niculita Hirzel H, Roulin A, Fuwagalli L, unpublished data). In A. noctua, G. passerinum, O. scops, and S. aluco, where only one gene was amplified with these primers, the forward primer was replaced by a primer designed from conserved sites in exon 1 of the other owl species (Sfex1F; 5' -CAC TGG TGG TGC TGG GAG CC-3' ). PCRs were carried out in a final volume of $50 \mu \mathrm{l}$ containing $1 \times$ buffer Gold, $2.0 \mathrm{mM} \mathrm{MgCl} 2,0.2 \mathrm{mM}$ deoxynucleoside triphosphate, $0.5 \mu \mathrm{M}$ each primers, and $2 \mathrm{U}$ AmpliTaq Gold (Applied Biosystems, Foster City, CA). PCR conditions included an initial denaturation step at $95^{\circ} \mathrm{C}$ for $5 \mathrm{~min}, 35-40$ cycles of denaturation at $95{ }^{\circ} \mathrm{C}$ for $1 \mathrm{~min}$, primer-specific annealing temperature (Tyal-ex $1 \mathrm{~F}, 65^{\circ} \mathrm{C}$; Sf-ex $1 \mathrm{~F}, 62{ }^{\circ} \mathrm{C}$ ) for $1 \mathrm{~min}$, and primer extension at $72{ }^{\circ} \mathrm{C}$ for $3 \mathrm{~min}$. A final step at $72{ }^{\circ} \mathrm{C}$ for 7 min was used to complete primer extension. All the PCR products were run on a $1 \%$ agarose gel. The bands of expected length were excised and purified using the QIAquick Gel Extraction Kit (Qiagen). The purified products were cloned using the pGEM-T Easy Vector System (Promega, Dübendorf, Switzerland), and 10 clones per species were sequenced. To guard against heteroduplexed sequences that can be formed during PCR and to avoid artificial alleles produced by PCR errors, we performed a second reverse transcriptase-polymerase chain reaction (RT-PCR) and PCR for each species and sequenced an additional 10 clones obtained from these products. In further analyses, we considered only sequences that were found in both of the independent manipulations and a few sequences that could be confirmed by the sequence of the uncloned PCR products and that differed considerably from redundant sequences (following Edwards et al. 1995).

\section{Data Analysis}

Sequences were assembled in MEGA 4.0 (Tamura et al. 2007) and aligned using the ClustalW algorithm. Nucleotide diversity $(\pi)$ was calculated in Arlequin 3.0 (Excoffier et al. 2005). Phylogenetic relationships among owl MHCIIB sequences were reconstructed for exon 2 and exon 3 separately. For analyses on exon 3, only one of the highly similar sequences per species was used. To reconstruct the evolutionary relationships of owl MHCIIB sequences with that of other bird species, we used 26 avian MHCIIB sequences from GenBank that contained exons 2 and 3. Corresponding sequences of spectacled caiman (Caiman crocodilus) and tuatara (Sphenodon punctatus) MHCIIB were added as outgroups. Phylogenetic reconstructions of the avian MHCIIB were performed on each exon separately and on a combined matrix. Prior to phylogenetic analysis, we tested for the loss of information in our data due to substitution saturation using the $\mathrm{I}_{\mathrm{SS}}$ index by Xia et al. (2003) in DAMBE (Xia and Xie 2001). To evaluate nucleotide substitution models, we used MrModeltest 2.2 (Nylander 2004). Best models were selected following the Akaike information criterion (Akaike 1974). Based on these selected substitution models, phylogenetic trees were constructed using MrBayes 3.1 (Ronquist and Huelsenbeck 2003) and the maximum likelihood (ML) method in RaxML (Stamatakis 2006). The most likely Bayesian topologies for owl exon 2 and avian exon 3 were tested against alternative phylogenetic hypotheses using ShimodairaHasegawa (SH) tests (Shimodaira and Hasegawa 1999) in PAUP (Swofford 2003). For owl exon 2, the inferred topology was compared with topologies with species-specific grouping of alleles, that is, with 1) the cytochrome $b$ tree, 2) a user-made tree where the topology of the inferred tree was changed to a tree with only species-specific clusters by minimal moving of branches of the inferred topology, and 3) with a topology where transspecies polymorphism was restricted to the genera (supplementary fig. S1a-c, Supplementary Material online). The cytochrome $b$ tree used in this analysis was constructed in MrBayes 3.1 with the best nucleotide substitution model determined by MrModeltest 
Table 1

Sample and Locus Information

\begin{tabular}{|c|c|c|c|c|}
\hline \multirow[b]{2}{*}{ Family } & \multirow[b]{2}{*}{ Species } & \multirow{2}{*}{$\begin{array}{c}\text { Species' } \\
\text { Gene Prefix }\end{array}$} & \multicolumn{2}{|c|}{ Alleles by Locus } \\
\hline & & & $D A B 1$ & $D A B 2$ \\
\hline \multirow[t]{12}{*}{ Strigidae } & Tengmalm's owl, Aegolius funereus & Aefu & $* 01$ & $* 01$ \\
\hline & Short-eared owl, Asio flammeus & Asfl & $* 01$ & $* 01$ \\
\hline & Long-eared owl, Asio otus & Asot & $* 01, * 02$ & $* 01$ \\
\hline & Little owl, Athene noctua & Atno & $* 01, * 02$ & $* 01$ \\
\hline & Spotted eagle owl, Bubo africanus & Buaf & $* 01, * 02$ & $* 01$ \\
\hline & Snowy owl, Bubo scandiacus & Busc & $* 01, * 02$ & $* 01$ \\
\hline & Eurasian pygmy owl, Glaucidium passerinum & Glpa & $* 01, * 02$ & - \\
\hline & European scops owl, Otus scops & Otsc & $* 01$ & $* 01, * 02$ \\
\hline & Tawny owl, Strix aluco & Stal & $* 01, * 02$ & $* 01$ \\
\hline & Great gray owl, Strix nebulosa & Stne & $* 01$ & $* 01$ \\
\hline & Ural owl, Strix uralensis & Stur & $* 01$ & $* 01$ \\
\hline & Northern hawk owl, Surnia ulula & Suul & $* 01, * 02$ & $* 01, * 02, * 03$ \\
\hline Tytonidae & Barn owl, Tyto alba & Tyal & $* 01-* 04$ & $* 01, * 02$ \\
\hline
\end{tabular}

NOTE. -4 individuals have been cloned for barn owl, but only 1 was used in analyses of this study.

2.2 general time-reversible $(\mathrm{GTR}+\mathrm{G})$ using sequences (1,041 bp) of Wink and Heinrich (1999) retrieved from GenBank. For avian exon 3, the inferred topology was compared with phylogenetic hypotheses with songbirds (Passeriformes) and land- and waterfowl (Galloanseres) clustering outside a monophyletic owl (Strigiformes) cluster: 1) Strigiformes (Passeriformes, Galloanseres), 2) Passeriformes (Strigiformes, Galloanseres), 3) Galloanseres (Strigiformes, Passeriformes), and with 4) the molecular taxonomy of the orders as proposed by Ericson et al. (2006) and Brown et al. (2007) (supplementary fig. S1d-g, Supplementary Material online). To examine phylogenetic relationships based on neutral genetic variation, the numbers of synonymous substitutions per synonymous site $\left(d_{\mathrm{S}}\right)$ were estimated in CodeML (Yang and Nielsen 2000). Neighbor-Joining (NJ) trees were reconstructed in MEGA 4.0 (Tamura et al. 2007 ) based on these $d_{\mathrm{S}}$ values. Bootstrap tests of NJ phylogenies based on $d_{\mathrm{S}}$ calculated by the Nei-Gojobori method with Jukes-Cantor correction were conducted in MEGA 4.0 (Tamura et al. 2007), using 5,000 replicates.

Recombination analyses were performed using the RDP 3 beta 22 package (Martin et al. 2005). In a first run, the RDP (Martin and Rybicki 2000), GENECONV (Padidam et al. 1999), Maximum Chi (Smith 1992), and BootScan (Salminen et al. 1995) methods were applied using the default settings with a maximal $P$ value of 0.05 and the Bonferroni correction for multiple comparisons and the "disentangle overlapping signals" option set "on." The recombination events detected by at least 3 methods were then rechecked with all available methods. Only break points confirmed after this procedure were considered as determined. Estimates of recombination rates along the owl MHCIIB alignment were obtained with the INTERVAL program (McVean et al. 2002) implemented in the RDP package. An initial alignment-wide population scaled recombination rate (rho) of 10 was applied as a starting point. The estimated rho value was then used in final estimates of recombination rates per base pair. Although recombination and gene conversion are mechanistically different processes, the resulting pattern of polymorphism is similar in sequences of limited length (Wiehe et al. 2000;
Richman et al. 2003; Chen et al. 2007). We therefore do not discriminate between these processes and hereafter refer to them as recombination in the broad sense (Parham and Ohta 1996; Martinsohn et al. 1999).

\section{Results}

Isolation of MHCIIB in Owls

Making use of an RT-PCR-based approach, we amplified the whole of exon 2 (270 bp) and about $220 \mathrm{bp}$ of exon 3 of MHCIIB genes in 14 species of owls. Cloning resulted in 43 confirmed MHCIIB sequences, giving between 2 and 5 confirmed sequences per species and up to 2 alleles per locus (see below, table 1). In all, 210 polymorphic sites were identified, of which $165(79 \%)$ are situated in exon 2 and $45(21 \%)$ in exon 3. Eighty-eight percent of nonconserved antigen-binding sites (Brown et al. 1993) are polymorphic. Overall nucleotide diversity $(\pi)$ is $0.11 \pm 0.05$, of which $0.13 \pm 0.06$ fall into exon 2 , with most diversity located in antigen-binding sites $(\pi=0.28 \pm 0.14)$. Exon 3 exhibits less nucleotide diversity $(\pi=0.06 \pm 0.03)$. Overall, the level and distribution of polymorphism are consistent with predictions for functional MHC genes, and all owl sequences contain features expected for classical MHCIIB molecules (supplementary fig. S2, Supplementary Material online), such as the intradomain cysteine salt bridges, the conserved glycosylation site (NGT), conserved residues of the $\beta 1$ domain (Brown et al. 1993; Kaufman et al. 1994), and also highly conserved residues involved in CD4 binding in the human DRB molecule (Wang et al. 2001).

\section{Phylogenetic Reconstructions of Owl MHCIIB}

Phylogenetic relationships between owl MHCIIB sequences were reconstructed for exon 2 and exon 3 separately. This partitioning was chosen to detect differences with respect to the evolutionary histories of these functionally distinct regions. Reconstructions of phylogenetic relationships of owl MHCIIB are congruent between Bayesian and ML methods and reveal distinct patterns for exon 2 and 

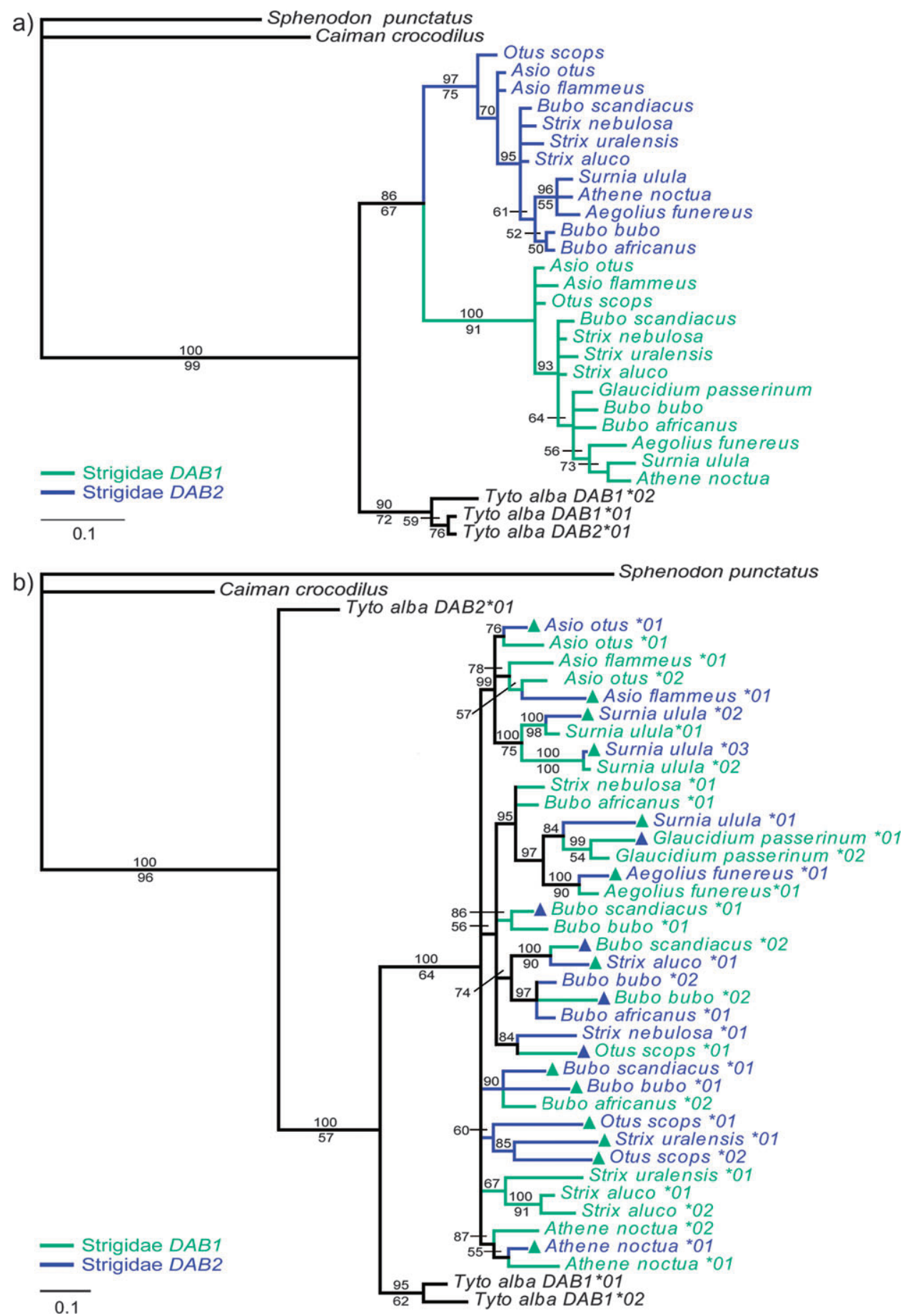

FIG. 1.-Consensus Bayesian topology (50\% majority consensus) and branch length for (a) owl exon 3 and (b) owl exon 2 sequences (GTR + G). Trees have been rooted with Sphenodon punctatus. Two runs and 4 chains per run were run for $5 \times 10^{6}$ generations. Numbers above and below branches indicate Bayesian and ML (1,000 bootstrap replicates) support, respectively. Only supports larger than 50 are shown. Triangle symbols in figure $1 b$ indicate recombinant alleles given in table 3 . Triangle color denotes the locus identity of the estimated exon 2 parental sequence.

exon 3 (fig. 1). Substitution saturation did not impact phylogenetic inference of MHCIIB, as tested by $\mathrm{I}_{\mathrm{SS}}$ statistics (all $P<0.05$ ).

Contrary to expectations from previous avian MHC phylogenies, where sequences cluster by species (Hess and Edwards 2002), phylogenetic reconstructions from owl exon 3 reveal 2 highly supported gene-specific clusters (fig. 1a). This topology remains unchanged when only synonymous substitutions $\left(d_{\mathrm{S}}\right)$ are considered (supplementary fig. S3a, Supplementary Material online). Both clusters contain sequences from all Strigidae species, except Eurasian pygmy owl (G. passerinum), which is found in only one cluster. Visual inspection of the alignments shows that sequences are highly similar within clusters but differ 


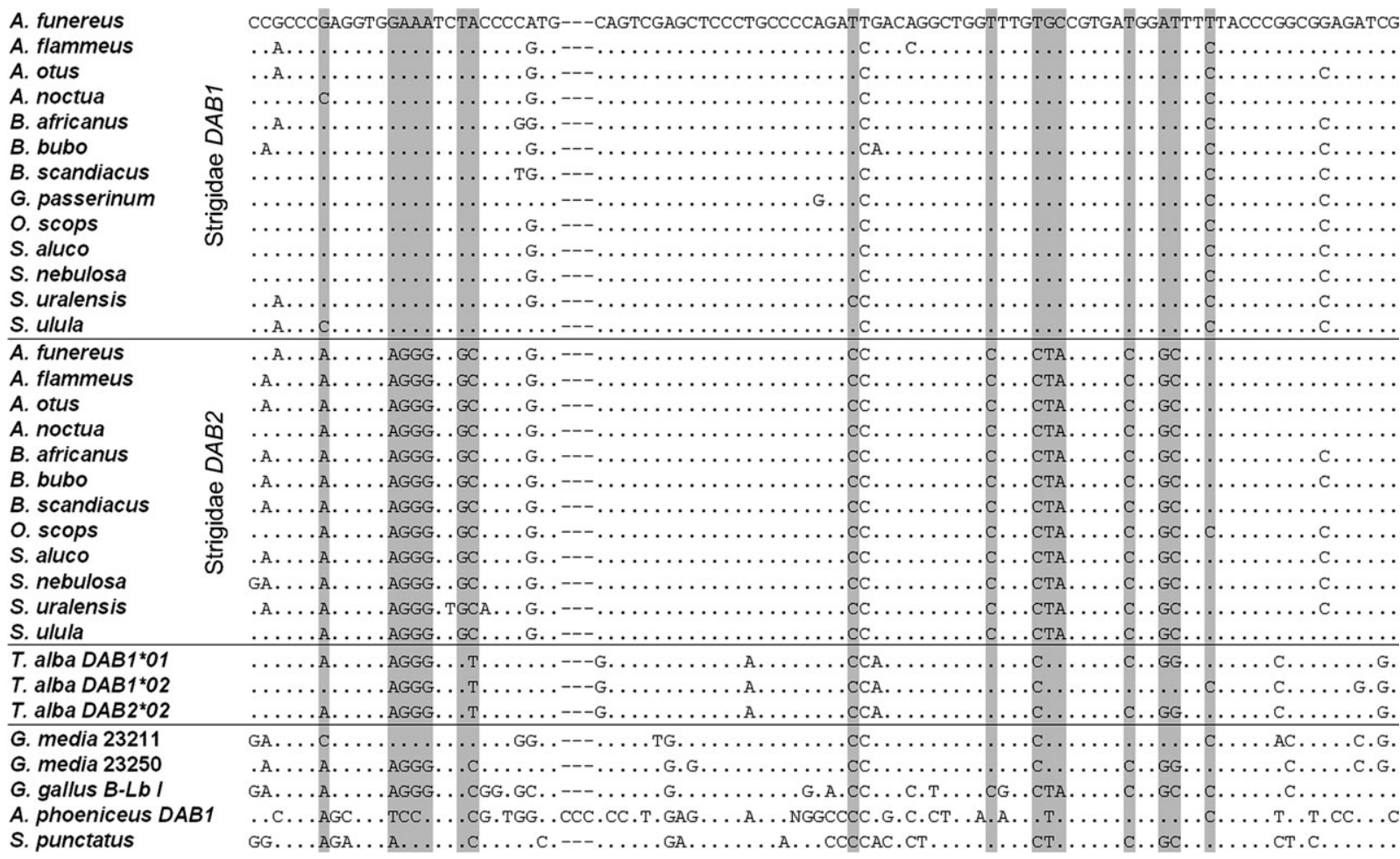

FIG. 2.-Nucleotide sequence alignment of the first 100-nt sites of MHCIIB exon 3 of owls, great snipe (Gallinago media), chicken (Gallus gallus) $B-L b I$, red-winged blackbird (Agelaius phoeniceus) DAB1, and tuatara (Sphenodon punctatus). Sites located in the locus signatures distinguishing Strigidae $D A B 1$ and $D A B 2$ are highlighted in gray.

among clusters in 11 amino acid residues (16-nt sites) located near the $5^{\prime}$ end of exon 3 (fig. 2). In these 11 residues, 8 amino acid substitutions are radical (supplementary fig. S4a, Supplementary Material online) (Zhang 2000). Based on the following arguments, we concluded that there are 2 genes that can be unambiguously distinguished upon the exon 3 sequence signatures: 1) high support of the clusters, 2) presence of all but one species in both clusters, and 3 ) the number of isolated sequences exceeding 2 per individual in 8 of 13 Strigidae species. Following the nomenclature of Klein et al. (1990), the genes were named $D A B I$ and $D A B 2$, preceded by a species prefix (table 1 ).

Phylogenetic reconstructions from exon 2 reveal 2 patterns that differ from those observed for exon 3 (fig. 1). First, the tree shows that exon 2 sequences are intermingled between genes even in highly supported intraspecific clusters, suggesting shuffling of exon 2 among genes. Second, for most species, at least one allele clusters with alleles from other species (fig. 1b). This pattern also occurs if reconstructions are based on synonymous substitutions $\left(d_{\mathrm{S}}\right)$ (supplementary fig. S3b, Supplementary Material online). Alleles of the genera Bubo and Strix are especially mixed between species and genera. For A. funereus, A. noctua, and G. passerinum, all alleles cluster together. SH tests show that the inferred topology has a significantly higher likelihood than a priori hypotheses with only intraspecific grouping of alleles or with transspecies polymorphism restricted to genera (table 2).

Barn owl (T. alba, Tytonidae) forms a separate cluster in phylogenetic reconstructions of both exons. For exon 3, this results from the virtual absence of variability between barn owl $D A B 1$ and $D A B 2$. The only differences (at 6-nt sites) among barn owl genes were found in the second allele of barn owl DABI (Tyal-DABI*02). Examination of the locus signature positions revealed that the barn owl $D A B$ consists of a compound of Strigidae $D A B$ signatures (fig. 2). In conjunction with the fact that 5 of the 6 polymorphic sites in barn owl exon 3 reside in the Strigidae $D A B$ signature residues and correspond to the respective Strigidae sequence, this unambiguously indicates orthology of the barn owl and Strigidae genes.

\section{Recombination in the Owl MHCIIB}

Recombination is assumed to play an important role in the evolution of the avian MHC. Although we obtained MHCIIB sequences from only one individual per species, estimates of recombination for our data set can be obtained by assuming that recombination could have taken place in ancestral species, which seems likely for MHC genes. Making use of an array of methods that detect recombination, we obtained evidence for 7 recombination events in owl MHCIIB data, resulting in a total of 18 recombinant sequences (table 3 , fig. $1 b$ ). All detected events concern interlocus recombination, that is, recombination between $D A B 1$ and $D A B 2$ sequences. Taking into account recombination in the exon 2 tree, by considering the locus identity of parental sequences of exon 2 instead of the locus identity designated by exon 3,11 of the 14 external nodes group sequences from the same locus, compared with only 4 when 
Table 2

Statistical Comparison of Phylogenetic Hypotheses for Owl Exon 2 and Avian Exon 3 Phylogenies

\begin{tabular}{lccc}
\hline Topology & $-\ln \mathrm{L} \Delta-\ln \mathrm{L}$ & $\mathrm{P}$ \\
\hline Owl exon 2 tree & & & \\
$\quad$ Inferred topology & 3860 & Best & - \\
1. Cytochrome $b$ & 4021 & 161 & $<0.001^{*}$ \\
2. Minimal moving species-specific & 4018 & 158 & $<0.001^{*}$ \\
clustering & & & \\
$\quad$ Transspecies polymorphism & 3967 & 107 & $0.001^{*}$ \\
restricted to genera & & & \\
Avian exon 3 tree & 1854 & Best & - \\
$\quad$ Inferred topology & 1874 & 20 & 0.082 \\
1. Strigiformes (Passeriformes, Galloanseres) & 1874 & 20 & 0.081 \\
2. Passeriformes (Strigiformes, Galloanseres) & 1874 & 20 & 0.081 \\
3. Galloanseres (Strigiformes, Passeriformes) & 1874 \\
4. Molecular taxonomy of orders & 1976 & 22 & 0.100 \\
\hline
\end{tabular}

NOTE.-Tree topologies are shown in supplementary figure S1 (Supplementary Material online).

recombination is not considered (fig. $1 b$ ). Two recombination break points were determined. Both are situated close to the exon 2 I exon 3 boundary (see supplementary fig. S2, Supplementary Material online). Analysis of recombination rates along the sequence alignments reveals considerable evidence for recombination in exon 2 (fig. 3). Recombination rates drop significantly at the exon 2 I exon 3 boundary and are lowest in the region containing the signature of the Strigidae $D A B 1$ and $D A B 2$ loci.

\section{Phylogenetic Reconstructions of Avian MHCIIB}

In addition to the phylogenetic relationships of MHCIIB in owls, we investigated the relationship of the owl $D A B$ sequences with that of other avian taxa. As expected from analyses within owls (fig. 1a), Strigidae $D A B 1$ and $D A B 2$ group separately in both the analyses based on exon 3 exclusively (fig. 4) and in the analyses combining exon 2 and exon 3 sequence data (supplementary fig. S5a, Supplementary Material online). The reconstruction based on combined data reflects taxonomic

Table 3

Recombinant Sequences at the Owl $D A B 1$ and $D A B 2$ loci

\begin{tabular}{|c|c|c|}
\hline Recombinant Sequences $^{\mathrm{a}}$ & $\begin{array}{l}\text { Nucleotide } \\
\text { Break } \\
\text { Point }\end{array}$ & $\begin{array}{l}\text { Potential } \\
\text { Parental } \\
\text { Sequences }\end{array}$ \\
\hline Suul-DAB2*02, Suul-DAB2*03 & n.d. & Unknown, Suul-DAB1*01 \\
\hline Otsc-DAB $1^{*} 01$ & n.d. & Unknown, Asfl-DABl*01 \\
\hline $\begin{array}{l}\text { Bubu-DAB1*02, Bubu-DAB2*01, } \\
\text { Busc-DAB1*01, Busc-DAB1*02, } \\
\text { Glpa-DAB1*01 }\end{array}$ & n.d. & $\begin{array}{l}\text { Stal-DAB1*02, } \\
\text { Stal-DAB2*01 }\end{array}$ \\
\hline $\begin{array}{l}\text { Busc-DAB2*01, Otsc-DAB2*01, } \\
\text { Stal-DAB2*01, Stur-DAB2*01 }\end{array}$ & n.d. & $\begin{array}{l}\text { Stne-DAB2*01, } \\
\text { Atno-DAB1*01 }\end{array}$ \\
\hline $\begin{array}{l}\text { Asfl-DAB2*01, Asot-DAB2*01, } \\
\text { Atno-DAB2*01 }\end{array}$ & n.d. & $\begin{array}{l}\text { Stne-DAB2*01, } \\
\text { Asot-DAB1*02 }\end{array}$ \\
\hline Otsc-DAB2*02 & 266 & Suul-DAB2*01, unknown \\
\hline Aefu-DAB2*01, Suul-DAB2*01 & 275 & $\begin{array}{l}\text { Stne-DAB2*01, } \\
\text { Stne-DAB1*01 }\end{array}$ \\
\hline
\end{tabular}

NoTE.- n.d.: not determined.

a Species prefixes are listed in table 1.

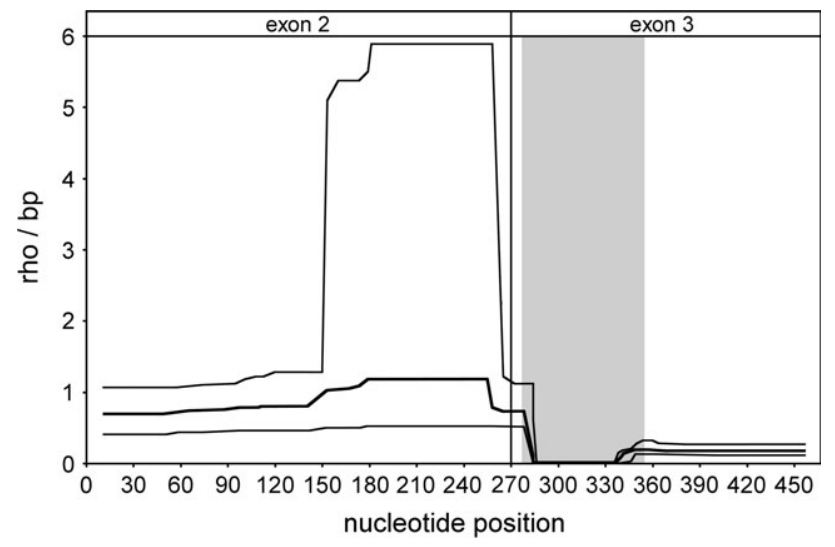

FIG. 3.-Recombination rates (rho) estimated along the owl MHCIIB nucleotide sequence alignment. The mean rho (bold line) per base pair and the upper and lower confidence intervals (narrow lines) are shown. The shaded area highlights the region containing the locus signatures (corresponding to nucleotide sites 7-84 in the alignment shown in fig. 2 and nucleotide sites 277-354 in supplementary fig. S2, Supplementary Material online).

relationships of the analyzed avian orders (supplementary fig. S5b, Supplementary Material online). In contrast, phylogenetic reconstructions based on exon 3 exclusively do not reveal a monophyletic owl gene clade but divide the avian MHCIIB into 2 clusters: songbirds (Passeriformes) group with Strigidae $D A B 1$, whereas land- and waterfowl (Galloanseres) and Humboldt's penguin (Spheniscus humboldti, Sphenisciformes) group with Strigidae DAB2 (fig. 4). Visual inspection of the sequence alignments shows that land- and waterfowl and Humboldt's penguin exhibit sequences highly similar to the $D A B 2$ signature, with 13 - to 14-nt sites out of 16 sites identical (fig. 2; supplementary fig. S4b, Supplementary Material online). Songbird exon 3 sequences however are more divergent from the owl MHCIIB, also with respect to the signature of $D A B 1$, with which they cluster in phylogenetic reconstructions. Sequences of the great snipe (Gallinagomedia, Charadriiformes) are placed together with those of the barn owl. Although this clustering is statistically not supported, examination of the great snipe's positions corresponding to the Strigidae $D A B$ signatures reveals that great snipe exhibits 2 types of exon 3 sequences. The great snipe sequences differ mainly at the same positions as Strigidae $D A B 1$ and $D A B 2$. At these positions, each sequence type contains a large part (10 out of 16-nt sites) of either the $D A B 1$ or $D A B 2$ signature (fig. 2). Due to mixed $D A B 1-D A B 2$ sequences at the locus signature residues (fig. 2), barn owl and great snipe sequences swap between the $D A B 1$ and $D A B 2$ clusters in phylogenetic analyses and consequently are placed outside the Strigidae $D A B$ clusters.

Statistical comparison of trees using SH tests revealed that the most likely topology for exon 3 is not significantly better than the a priori hypotheses that group the songbirds, land- and waterfowl orders basal to a monophyletic owl cluster, or the one following the molecular taxonomy of orders (table 2). Phylogenetic analyses based on synonymous substitutions $\left(d_{\mathrm{S}}\right)$ provide a different topology for exon 3 , with the positions of land- and waterfowl and songbirds changing (supplementary fig. S3c, Supplementary Material 


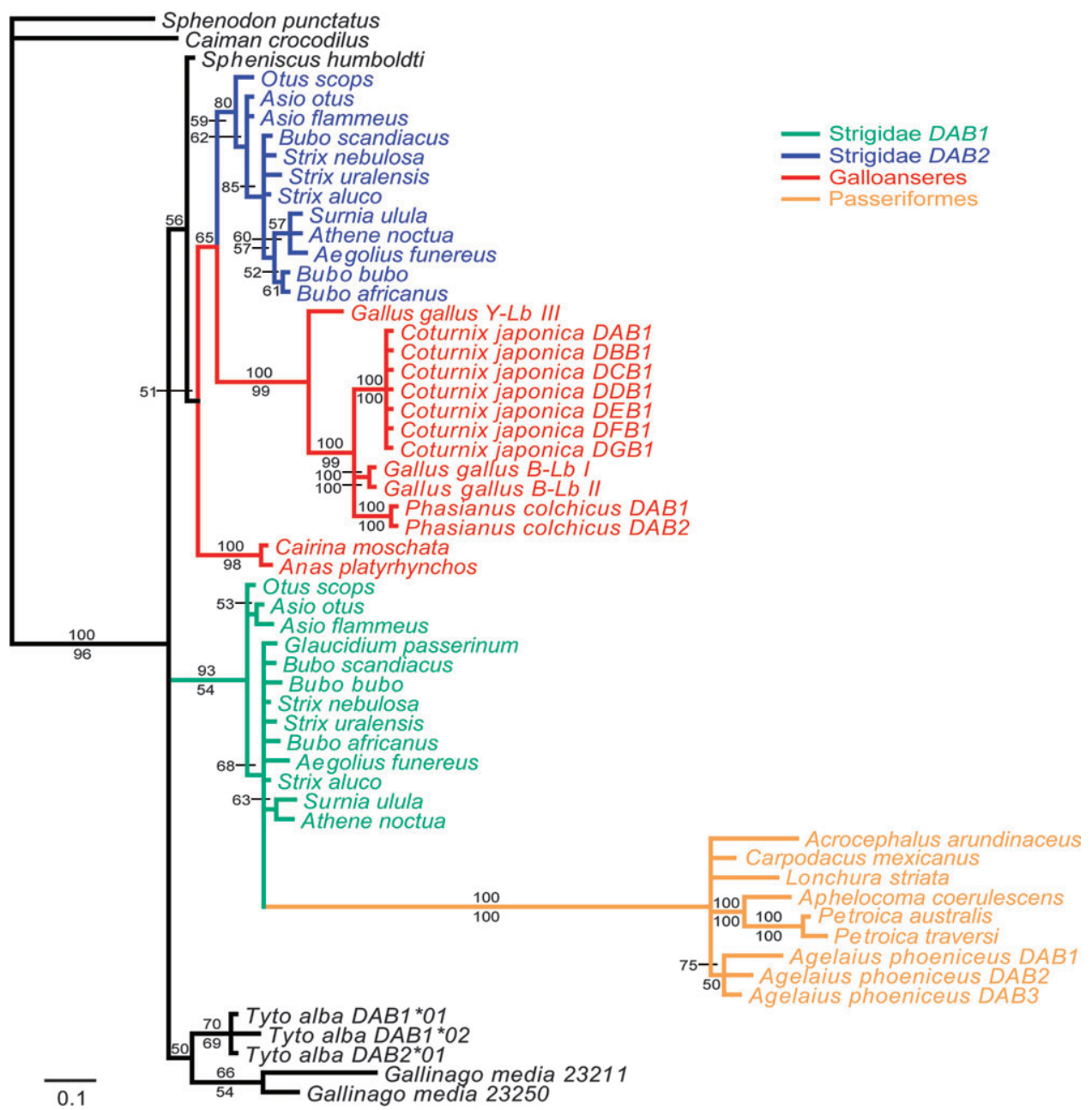

FIG. 4.-Consensus Bayesian topology (50\% majority consensus) and branch length for avian exon 3 sequences (GTR + G). The tree has been rooted with Sphenodon punctatus. Two runs and 4 chains per run were run for $5 \times 10^{6}$ generations. Numbers above and below branches indicate Bayesian and ML (1,000 bootstrap replicates) support, respectively. Only supports larger than 50 are shown. Accession numbers of analyzed species are as follows: S. punctatus, DQ124232; Caiman crocodilus, AF256652; Gallinago media, AF485404, AF485405, AF485408, and AF485412 (Ekblom et al. 2003); Spheniscus humboldti, AB154394; Anas platyrhynchos, DQ490139; Cairina moschata, DQ490138; Coturnix japonica, AB078884 (Shiina et al. 2004); Gallus gallus, AL023516, AB020221; Phasianus colchicus, AJ224349, AJ224348; Agelaius phoeniceus, AF030997, AF170972, and AF328738; Acrocephalus arundinaceus, AJ404376; Aphelocoma coerulescens, U23965; Carpodacus mexicanus, U23976; Lonchura striata, L42335; Petroica australis, AY428564; and Petroica traversi, AY428569.

online). On this tree, Strigidae $D A B 1$ and $D A B 2$ appear more closely related to each other than to the sequences of the other groups. However, the nodes in question are not statistically supported (supplementary fig. S3c, Supplementary Material online). This might be explained by the low frequency of synonymous substitutions in the analyzed sequences, presumably caused by the high GC saturation at third positions in exon $3(88-99 \%$, mean $=93.6 \pm 2.9 \%)$ (Satta et al. 1999).

\section{Discussion}

Evolutionary Patterns of the MHCIIB in Owls

Using an RT-PCR-based approach, we identified up to 3 functional MHCIIB genes in owls. Although extensive cloning might uncover additional gene copies, the genomic organization of the owl MHC resembles the simple organi- zation in nonpasserine species such as chicken (Zoorob et al. 1990; Kaufman et al. 1999). This finding is in line with the proposition of a simple MHC organization in birds of prey recently made by Alcaide et al. (2007). However, all loci identified in owls by these authors correspond to $D A B I$ loci reported here. No $D A B 2$-like sequences were identified, making it impossible for Alcaide et al. (2007) to detect the divergent evolution identified by our analyses.

The disparate patterns of evolution for the owl exon 2 versus exon 3 are consistent with the different function of these regions. Exon 2 encodes the antigen-binding region and exhibits transspecies evolution in some of the owl species. In contrast, exon 3 underwent divergent evolution, allowing gene duplication history to be traced back. Both transspecies evolution and divergent evolution are typical for the mammalian MHC. Although it is known that avian exon 2 sequences do not exclusively group within species (Vincek et al. 1997; Edwards et al. 1999; Bonneaud et al. 
2004), overall patterns of MHC evolution in Strigidae owls are rather comparable with that of primates (Kriener et al. 2001) or cyprinid fish (Ottová et al. 2005) and less so to what has previously been found in birds. Yet, studies in primates have shown that such transspecies-like patterns in MHCIIB exon 2 can arise by convergence (Kriener et al. 2000, 2001). In owls, phylogenetic reconstructions based on synonymous substitutions are in line with those from whole sequence information, indicating that the similarity between exon 2 sequences of different species is not due to convergence by natural selection (supplementary fig. S3b, Supplementary Material online). Transspecies evolution was detected in about two-thirds of the analyzed species, but it appears not to be the general rule in Strigidae. In some species, all exon 2 sequences cluster together, indicating concerted evolution. Comparison of the patterns of transspecies and concerted evolution to a species-level phylogeny and to divergence time estimates for owl species could provide information on the strength of historical balancing selection. Unfortunately, such data are lacking so far.

Given that in previous studies MHC loci cannot be distinguished by sequence data, the persistence of locus signatures in owl $D A B$ genes raises questions on how genomic organization or selection could prevent the homogenization of genes. So far, orthology among avian MHC genes has been detected from coding sequences in only black grouse and chicken (Strand et al. 2007). The independent evolution of $B-L b$ and $Y-L b$ orthologs in these species can be explained by genomic organization as the orthologs are situated in the unlinked $B$ and $Y$ loci (Briles et al. 1993; Shiina et al. 2006). Because the rates of interlocus recombination are expected to be inversely proportional to the distance between loci (e.g., Ezawa et al. 2006), unlinked loci like $B-L b$ and $Y-L b$ are expected to be less prone to homogenization by recombination. This might suggest that locus signatures in owl $D A B$ genes may have persisted because the loci are located apart from each other. Although our data do not allow us to draw precise conclusions on genomic organization of the owl MHCIIB, the existing information rather contradicts this hypothesis. The presence of interlocus recombination indicates that the 2 genes are linked (Ezawa et al. 2006). Alternative explanations for the persistence of distinct locus signatures invoke locus-specific selection. The radical amino acid changes (supplementary fig. S4a, Supplementary Material online) and the absence of recombination within the locus signature (fig. 3) might indicate that these genes exhibit functional differences and that changes in the signature sequences may be counteracted by natural selection. The differences are situated in the linkage domain. Two negative charged amino acids in $D A B 1$ (E and $\mathrm{D}$ ) are represented in $D A B 2$ by a positive amino acid $(\mathrm{R})$ and a neutral amino acid $(\mathrm{G})$, respectively (supplementary fig. S4a, Supplementary Material online). These differences in charges could result in a different 3 dimensional conformation of the protein.

Studies in diverse vertebrate lineages have convincingly shown that recombination can underpin diversification of the MHC (e.g., Andersson and Mikko 1995; Ohta 1999; Reusch and Langefors 2005; Bos and Waldman 2006). Recombination is assumed to be the major force in shaping the patterns of concerted evolution in the avian
MHC (Hess and Edwards 2002). Despite divergent evolution of Strigidae MHCIIB exon 3 sequences, our analyses suggest that recombination also played an important role in the evolution of Strigidae MHCIIB genes, resulting in concerted evolution of exon 2 in some owl species. Intermingling of sequences among genes, as observed for exon 2 in Strigidae owls (fig. 1b), is indicative of shuffling of exons among genes by recombination (Nei et al. 1997; Gu and Nei 1999). Similar translocations of alleles to different parts of the tree topology have also been observed in amphibians (Bos and Waldman 2006) and fish (Reusch and Langefors 2005). Indeed, our analyses reveal recombination in the owl MHC, with recombination rates highest in exon 2 (fig. 3). All recombination events were detected between rather than within genes. We stress however that the estimated parental sequences represent proxies of the sequences that contributed to recombination as the detected events must have taken place in ancestral species. Analyses of the owl exon 2 tree that takes into account recombination revealed that at least some of the external nodes containing sequences from different loci result from recombination (fig. $1 b$ ). The intermingling of exon 2 sequences among genes, as revealed in figure $1 b$, thus reflects recombination in ancestral owl species. This suggests that the generation of new alleles by recombination and especially shuffling of exon 2 among genes might still be of greater importance in owls than in most nonavian lineages.

\section{Early Divergence or Convergent Evolution in the Avian} MHC?

Given the peculiar phylogenetic patterns of owl MHCIIB, we were interested in the phylogenetic relationships of owl MHCIIB sequences to that of other avian species. The combined exon 2 and exon 3 avian MHCIIB data largely reflect the taxonomic relationships of the analyzed orders (supplementary fig. S5a, Supplementary Material online). However, exon 3 exhibits an intriguing pattern, with Strigidae sequences splitting the phylogeny in 2 major clades. SH tests and the $d_{\mathrm{S}}$ tree neither support this topology nor contradict it, taking into consideration that the SH test is very conservative, minimizing type I error (Buckley and Cunningham 2002; Shimodaira 2002), and that the $d_{\mathrm{S}}$ tree is statistically not supported at all. Although the $d_{\mathrm{S}}$ topology is similar to the order taxonomy except for Galloanseres (supplementary fig. S5b, Supplementary Material online), in particular, the clustering of Galloanseres with Strigidae $D A B 2$ is supported by the high similarity within the $D A B 2$ signature (fig. 2; supplementary fig. S4b, Supplementary Material online).

The peculiar phylogenetic relationships of avian MHCIIB exon 3 sequences could be explained either by 1) early divergence of the owl $D A B 1$ and $D A B 2$, predating at least the neoavian radiation and subsequent masking of orthology by concerted evolution or loss of one gene in some lineages as in the birth-and-death model of evolution or alternatively 2) by convergent evolution. It appears difficult to disentangle these 2 scenarios because the available sequence information is very limited. Convergent evolution would require a common selective pressure on a region outside the functionally distinct domains involved in 
antigen and T lymphocyte binding (supplementary fig. S2, Supplementary Material online). Interpretation of the avian exon 3 phylogeny in terms of convergent evolution emphasizes the hypothesis of functional divergence of Strigidae $D A B$ genes. If ancestral to Neoaves, the $D A B I$ and $D A B 2$ genes should also be found in songbirds and Humboldt's penguin. However, differences in the rate of concerted evolution among lineages (see also below) can explain the disappearance of the signs for orthology in one lineage but not in others. Likewise, birth-and-death evolution might have resulted in the loss of one gene in some lineages. If scenario (1) holds and the owl $D A B$ genes represent ancestral avian $\mathrm{MHC}$ lineages, extensive screening in basal songbird lineages as Tyrants (Tyrannidae) or Corvids (Corvoidea) might uncover genes orthologous to owl $D A B$ genes.

Implications for the Understanding of Avian MHC evolution

The phylogenetic relationships revealed in the owl MHCIIB challenge the patterns of MHC evolution demonstrated in avian lineages so far (Edwards et al. 1999; Hess and Edwards 2002). Whereas orthology in the avian MHC was previously reported in only 3 species pairs, we provide strong evidence for orthology of MHCIIB genes throughout the owl order. Moreover, orthology with owl MHCIIB genes can even be traced beyond the order's boundary back to great snipe. Based on divergence time estimates between Charadriiformes and Strigiformes (Brown et al. 2007), the origin of the $D A B 1$ and $D A B 2$ genes can be dated back to 83-122 MYA (see supplementary fig. S5b, Supplementary Material online). This is one order of magnitude longer than previous observations in the avian MHC, dating 4 MYA in Chatham Island robin and South Island robins (Campbell 1998), 20 MYA in chicken and pheasant (Helm-Bychowski and Wilson 1986), and 31-88 MYA in chicken and black grouse (Pereira and Baker 2006), especially for the first 2 cases, where evidence for orthology comes from noncoding sequences that experience less conversion than coding regions (Irwin and Wilson 1990; Wittzell et al. 1999). The orthologous relationship of owl MHC genes with great snipe suggests that our results are not restricted to owls but could represent a more general pattern. From this point of view, it appears questionable whether the taxa studied so far provide an adequate picture of avian MHC evolution.

Previous studies suggested that rounds of gene duplication and homogenization in avian MHCIIB occur on a faster timescale than in mammals, due to increased rates of concerted evolution in birds (Edwards et al. 1995; Wittzell et al. 1999). Our evidence from owls now challenges the generality of this conclusion. MHCIIB genes have apparently experienced more recombination in barn owls than in Strigidae owls, indicating that rates of concerted evolution differ between these sister lineages. Furthermore, barn owl genes are more homogenized than genes of the more distantly related great snipe (see supplementary fig. S5b, Supplementary Material online), suggesting that rates of concerted evolution may be independent of the lineage's divergence. We suggest that, contrary to what has been assumed so far, the relative impact of diversifying and ho- mogenizing forces on the evolution of the MHC might not only differ between birds and mammals but also varies considerably among birds. This suggestion has important implications for the reconstruction of the evolutionary history of the avian MHC. The divergence between orthologs was shown to increase with the rates of concerted evolution (Hurles et al. 2004). Variation in the rates of concerted evolution thereby might introduce variation in the degree of divergence of MHC genes among lineages, resulting in complex phylogenetic patterns, with orthology of genes detectable in some lineages but not in others. Such complex evolutionary dynamics could explain the high degree of divergence of songbird sequences (fig 2; supplementary fig. S5a, Supplementary Material online). The presence of multiple duplications as indicated in previous studies (Hess and Edwards 2002) might increase the rates of interlocus recombination in this lineage, which in turn can enhance the degree of divergence between orthologs and impede the detection of orthology with genes of other lineages. Alternatively, songbirds could simply have maintained a third, divergent ancestral gene copy that has been lost in the other lineages. As illustrated by this case and demonstrated for barn owl and great snipe genes, in some cases phylogenetic reconstructions alone do not suffice for the reconstruction of orthology under a scenario with high variation in evolutionary dynamics. Still, in many lineages, complete insight into the evolutionary history of the avian MHC might only be gained by information on genomic organization.

As recombination is assumed to play a major role in the evolution of the avian MHC (Garrigan and Edwards 1999; Hess and Edwards 2002), estimates of current as well as past recombination from owls might add an important contribution to the understanding of concerted evolution in the avian MHC. Our results highlight the importance of considering gene regions other than just the ones involved in antigen binding in order to gain a more comprehensive understanding of avian MHC evolution. As suggested by Edwards et al. (1999), sampling at different levels in the avian tree proves to be important. Future work implicating information from genomic sequences from a number of species will have to show to what extent evolutionary rates at the MHC vary among species and will shed light on the timescale at which diversification and homogenization in the avian MHC take place.

\section{Supplementary Material}

Supplementary figures S1-S5 are available at Molecular Biology and Evolution online (http://www.mbe. oxfordjournals.org/). The sequences reported in this paper have been deposited in the GenBank database (accession numbers: EF641223-EF641262).

\section{Acknowledgments}

We thank Pascal-Antoine Christin, Anne-Lyse Ducrest, Scott V. Edwards, Seraina Klopfstein, Patrik Nosil, Marc Robinson-Rechavi, Walter Salzburger, Ian Sanders, Naoko Takezaki, Jon Yearsley, and 4 anonymous reviewers for helpful comments on earlier versions of this paper. 
Steven Diethelm, the Zoo La Garenne, the Museum of Zoology Lausanne, and the Natural History Museum of Bern contributed samples. This study was supported by Swiss National Science Foundation grants 3100A0-109852/1 to L.F. and PPOOA-102913 to A.R.

\section{Literature Cited}

Akaike H. 1974. A new look at the statistical model identification. IEEE Trans Automat Contr. 19:716-723.

Alcaide M, Edwards S, Negro J. 2007. Characterization, polymorphism, and evolution of MHC class II B genes in birds of prey. J Mol Evol. 65:541-554.

Andersson L, Mikko S. 1995. Generation of MHC class II diversity by intra- and intergenic recombination. Immunol Rev. 143:5-12.

Beck S, Trowsdale J. 2000. The human major histocompatibility complex: lessons from the DNA sequence. Annu Rev Genomics Hum Genet. 1:117-137.

Bernatchez L, Landry C. 2003. MHC studies in nonmodel vertebrates: what have we learned about natural selection in 15 years? J Evol Biol. 16:363-377.

Bonneaud C, Sorci G, Morin V, Westerdahl H, Zoorob R, Wittzell H. 2004. Diversity of $M h c$ class I and IIB genes in house sparrows (Passer domesticus). Immunogenetics. 55:855-865.

Bos DH, Waldman B. 2006. Evolution by recombination and transspecies polymorphism in the MHC class I gene of Xenopus laevis. Mol Biol Evol. 23:137-143.

Briles WE, Goto RM, Auffray C, Miller MM. 1993. A polymorphic system related to but genetically independent of the chicken major histocompatibility complex. Immunogenetics. 37:408-414.

Brown JH, Jardetzky TS, Gorga JC, Stern LJ, Urban RG, Strominger JL, Wiley DC. 1993. Three-dimensional structure of the human class II histocompatibility antigen HLA-DR1. Nature. 364:33-39.

Brown JW, Payne RB, Mindell DP. 2007. Comment. Nuclear DNA does not reconcile 'rocks' and 'clocks' in Neoaves: a comment on Ericson et al. Biol Lett. 3:257-259.

Buckley TR, Cunningham CW. 2002. The effects of nucleotide substitution model assumptions on estimates of nonparametric bootstrap support. Mol Biol Evol. 19:394-405.

Campbell HJ. 1998. Fauna and flora of the Chatham Islands: less than 4 my old? Geology and genes. In: Cooper RA, Jones C, editors. Geological Society of New Zealand: Wellington (New Zealand). p. 15-16

Chen J-M, Cooper DN, Chuzhanova N, Ferec C, Patrinos GP. 2007. Gene conversion: mechanisms, evolution and human disease. Nat Rev Genet. 8:762-775.

Doherty PC, Zinkernagel RM. 1975. Enhanced immunological surveillance in mice heterozygous at the $\mathrm{H}-2$ gene complex. Nature. 256:50-52.

Edwards SV, Grahn M, Potts WK. 1995. Dynamics of Mhc evolution in birds and crocodilians: amplification of class II genes with degenerate primers. Mol Ecol. 4:719-729.

Edwards SV, Hess CM, Gasper J, Garrigan D. 1999. Toward an evolutionary genomics of the avian Mhc. Immunol Rev. 167:119-132.

Edwards SV, Wakeland EK, Potts W. 1995. Contrasting histories of avian and mammalian Mhc genes revealed by class II B sequences from songbirds. Proc Natl Acad Sci USA. 92:12200-12204.

Ekblom R, Grahn M, Höglund J. 2003. Patterns of polymorphism in the MHC class II of a non-passerine bird, the great snipe (Gallinago media). Immunogenetics. 54:734-741.
Ericson P, Anderson C, Britton T, Elzanowski A, Johansson U, Källersjö M, Ohlson J, Parsons T, Zuccon D, Mayr G. 2006. Diversification of Neoaves: integration of molecular sequence data and fossils. Biol Lett. 2:543-547.

Excoffier L, Laval G, Schneider S. 2005. Arlequin (version 3.0): an integrated software for population genetics data analysis. Evol Bioinform Online. 1:47-50.

Ezawa K, Oota S, Saitou N. 2006. Genome-wide search of gene conversions in duplicated genes of mouse and rat. Mol Biol Evol. 23:927-940.

Figueroa F, Gunther E, Klein J. 1988. MHC polymorphism predating speciation. Nature. 335:265-267.

Garrigan D, Edwards SV. 1999. Polymorphism across an exonintron boundary in an avian Mhe class II B gene. Mol Biol Evol. 16:1599-1606.

Geraghty DE, Daza R, Williams LM, Vu Q, Ishitani A. 2002. Genetics of the immune response: identifying immune variation within the $\mathrm{MHC}$ and throughout the genome. Immunol Rev. 190:69-85.

Gu X, Nei M. 1999. Locus specificity of polymorphic alleles and evolution by a birth-and-death process in mammalian MHC genes. Mol Biol Evol. 16:147-156.

Helm-Bychowski KM, Wilson AC. 1986. Rates of nuclear DNA evolution in pheasant-like birds: evidence from restriction maps. Proc Natl Acad Sci USA. 83:688-692.

Hess CM, Edwards SV. 2002. The evolution of the major histocompatibility complex in birds. Bioscience. 52: 423-431.

Hughes AL, Yeager M. 1998. Natural selection at major histocompatibility complex loci of vertebrates. Annu Rev Genet. 32:415-435.

Hurles M, Willey D, Matthews L, Hussain SS. 2004. Origins of chromosomal rearrangement hotspots in the human genome: evidence from the AZFa deletion hotspots. Genome Biol. 5:R55.

Irwin DM, Wilson AC. 1990. Concerted evolution of ruminant stomach lysozymes. Characterization of lysozyme cDNA clones from sheep and deer. J Biol Chem. 265:4944-4952.

Jarvi SI, Tarr CL, McIntosh CE, Atkinson CT, Fleischer RC. 2004. Natural selection of the major histocompatibility complex (Mhc) in Hawaiian honeycreepers (Drepanidinae). Mol Ecol. 13:2157-2168.

Kaufman J, Milne S, Gobel TWF, Walker BA, Jacob JP, Auffray C, Zoorob R, Beck S. 1999. The chicken B locus is a minimal essential major histocompatibility complex. Nature. 401:923-925.

Kaufman J, Salomonsen J, Flajnik M. 1994. Evolutionary conservation of MHC class I and class II molecules-different yet the same. Semin Immunol. 6:411-424.

Kelley J, Walter L, Trowsdale J. 2005. Comparative genomics of major histocompatibility complexes. Immunogenetics. 56: 683-695.

Kikkawa EF, Tsuda TT, Naruse TK, et al. (12 co-authors). 2005. Analysis of the sequence variations in the $M h c D R B l$-like gene of the endangered Humboldt penguin (Spheniscus humboldti). Immunogenetics. 57:99-107.

Klein J, et al. 1986. Natural history of the major histocompatibility complex. New York: Wiley.

Klein J, Bontrop R, Dawkins RL, Erlich HA, Gryllensten UB, Heise ER, Jones PP, Parham P, Wakeland EK, Watkins DI. 1990. Nomenclature for major histocompatibility complexes of different species: a proposal. Immunogenetics. 31: 217-219.

Klein J, Sato A, Nagl S, O’hUigin C. 1998. Molecular transspecies polymorphism. Annu Rev Evol Syst. 29:1-21.

Kriener K, O'hUigin C, Tichy H, Klein J. 2000. Convergent evolution of major histocompatibility complex molecules in 
humans and New World monkeys. Immunogenetics. 51:169-178.

Kriener K, O'hUigin C, Klein J. 2001. Independent origin of functional MHC class II genes in humans and new world monkeys. Hum Immunol. 62:1-14.

Kulski JK, Shiina T, Anzai T, Kohara S, Inoko H. 2002. Comparative genomic analysis of the MHC: the evolution of class I duplication blocks, diversity and complexity from shark to man. Immunol Rev. 190:95-122.

Lawlor DA, Ward FE, Ennis PD, Jackson AP, Parham P. 1988. HLA-A and B polymorphisms predate the divergence of humans and chimpanzees. Nature. 335:268-271.

Martin D, Rybicki E. 2000. RDP: detection of recombination amongst aligned sequences. Bioinformatics. 16: 562-563.

Martin DP, Williamson C, Posada D. 2005. RDP2: recombination detection and analysis from sequence alignments. Bioinformatics. 21:260-262.

Martinsohn JT, Sousa AB, Guethlein LA, Jonathan C, Howard JC. 1999. The gene conversion hypothesis of MHC evolution: a review. Immunogenetics. 50:168-200.

McVean G, Awadalla P, Fearnhead P. 2002. A coalescent-based method for detecting and estimating recombination from gene sequences. Genetics. 160:1231-1241.

Miller HC, Lambert DM. 2004. Gene duplication and gene conversion in class II MHC genes of New Zealand robins (Petroicidae). Immunogenetics. 56:178-191.

Nei M, Gu X, Sitnikova T. 1997. Evolution by the birth-and-death process in multigene families of the vertebrate immune system. Proc Natl Acad Sci USA. 94: 7799-7806.

Nei M, Hughes AL. 1992. Balanced polymorphism and evolution by the birth-and-death process in the MHC loci. In: Tsuji K, Aizawa M, Sasazuki T, editors. 11th Histocompatibility Workshop and Conference. November 1991. Oxford (UK): Oxford University Press. p. 27-38.

Nei M, Rooney AP. 2005. Concerted and birth-and-death evolution of multigene families. Annu Rev Genet. 39:121-152.

Nylander JAA. 2004. MrModeltest v2. Evolutionary Biology Centre: Uppsala University Uppsala, Sweden

Ohta T. 1999. Effect of gene conversion on polymorphic patterns at major histocompatibility complex loci. Immunol Rev. 167:319-325.

Ottová E, Šimkováa A, Martin J-F, de Bellocq JG, Gelnar M, Allienne J-F, Morand S. 2005. Evolution and trans-species polymorphism of MHC class II $\beta$ genes in cyprinid fish. Fish Shellfish Immunol. 18:199-222.

Padidam M, Sawyer S, Fauquet CM. 1999. Possible emergence of new geminiviruses by frequent recombination. Virology. 265:218-225.

Parham P, Ohta T. 1996. Population biology of antigen presentation by MHC class I molecules. Science. 272: 67-74.

Penn DJ, Damjanovich K, Potts WK. 2002. MHC heterozygosity confers a selective advantage against multiple-strain infections. Proc Natl Acad Sci USA. 99:11260-11264.

Pereira SL, Baker AJ. 2006. A molecular timescale for galliform birds accounting for uncertainty in time estimates and heterogeneity of rates of DNA substitutions across lineages and sites. Mol Phylogenet Evol. 38:499-509.

Reusch T, Langefors Å. 2005. Inter- and intralocus recombination drive MHC class IIB gene diversification in a Teleost, the three-spined stickleback Gasterosteus aculeatus. J Mol Evol. 61:531-541.

Richman AD, Herrera LG, Deanna N, Schierup MH. 2003. Relative roles of mutation and recombination in generating allelic polymorphism at an MHC class II locus in Peromyscus maniculatus. Genet Res. 82:89-99.

Ronquist F, Huelsenbeck JP. 2003. MrBayes 3: Bayesian phylogenetic inference under mixed models. Bioinformatics. 19:1572-1574.

Salminen MO, Carr JK, Burke DS, McCutchan FE. 1995. Identification of breakpoints in intergenotypic recombinants of HIV type- 1 by bootscanning. AIDS Res Hum Retroviruses. 11:1423-1425.

Satta Y, Kupfermann H, Li Y-J, Takahata N. 1999. Molecular clock and recombination in primate Mhc genes. Immunol Rev. 167:367-379.

Shiina T, Hosomichi K, Hanzawa K. 2006. Comparative genomics of the poultry major histocompatibility complex. Anim Sci J. 77:151-162.

Shiina T, Shimizu S, Hosomichi K, Kohara S, Watanabe S, Hanzawa K, Beck S, Kulski JK, Inoko H. 2004. Comparative genomic analysis of two avian (quail and chicken) MHC regions. J Immunol. 172:6751-6763.

Shimodaira H. 2002. An approximately unbiased test of phylogenetic tree selection. Syst Biol. 51:492-508.

Shimodaira H, Hasegawa M. 1999. Multiple comparisons of loglikelihoods with applications to phylogenetic inference. Mol Biol Evol. 16:1114-1116.

Smith JM. 1992. Analyzing the mosaic structure of genes. J Mol Evol. 34:126-129.

Stamatakis A. 2006. RAxML-VI-HPC: maximum likelihoodbased phylogenetic analyses with thousands of taxa and mixed models. Bioinformatics. 22:2688-2690.

Strand T, Westerdahl H, Höglund J, Alatalo RV, Siitari H. 2007. The Mhc class II of the Black grouse (Tetrao tetrix) consists of low numbers of B and Y genes with variable diversity and expression. Immunogenetics. 59:725-734.

Swofford DL. 2003. PAUP*: phylogenetic analysis using parsimony (*and other methods). Version 4. Sunderland (MA): Sinauer Associates.

Tamura K, Dudley J, Nei M, Kumar S. 2007. MEGA4: molecular evolutionary genetics analysis (MEGA) software version 4.0. Mol Biol Evol. 24:1596-1599.

Tsuda T, Tsuda M, Naruse T, et al. (11 co-authors). 2001. Phylogenetic analysis of penguin (Spheniscidae) species based on sequence variation in MHC class II genes. Immunogenetics. 53:712-716.

Vincek V, O'hUigin C, Satta Y, Takahata N, Boag PT, Grant PR, Grant BR, Klein J. 1997. How large was the founding population of Darwin's finches? Proc R Soc Lond B Biol Sci. 264:111-118.

Wang J-h, Meijers R, Xiong Y, Liu J-h, Sakihama T, Zhang R, Joachimiak A, Reinherz EL. 2001. Crystal structure of the human CD4 N-terminal two-domain fragment complexed to a class II MHC molecule. Proc Natl Acad Sci USA. 98:10799-10804.

Wiehe T, Mountain J, Parham P, Slatkin M. 2000. Distinguishing recombination and intragenic gene conversion by linkage disequilibrium patterns. Genet Res. 75:61-73.

Wink M, Heinrich P. 1999. Molecular evolution and systematics of owls (Strigiformes). In: König C, Weick F, Beckin JH, editors. Owls of the world. Mountfield (UK): Pica Press. p. 39-57.

Wittzell H, Bernot A, Auffray C, Zoorob R. 1999. Concerted evolution of two Mhc class II B loci in pheasants and domestic chickens. Mol Biol Evol. 16:479-490.

Wittzell H, Madsen T, Westerdahl H, Shine R, von Schantz T. 1998. MHC variation in birds and reptiles. Genetica. 104:301-309.

Xia X, Xie Z. 2001. DAMBE: data analysis in molecular biology and evolution. J Hered. 92:371-373. 
Xia X, Xie Z, Salemi M, Chen L, Wang Y. 2003. An index of substitution saturation and its application. Mol Phylogenet Evol. 26:1-7.

Yang Z, Nielsen R. 2000. Estimating synonymous and nonsynonymous substitution rates under realistic evolutionary models. Mol Biol Evol. 17:32-43.

Zhang J. 2000. Rates of conservative and radical nonsynonymous nucleotide substitutions in mammalian nuclear genes. J Mol Evol. 50:56-68.
Zoorob R, Béhar G, Kroemer G, Auffray C. 1990. Organisation of a functional chicken class II $B$ gene. Immunogenetics. 31:179-187.

Naoko Takezaki, Associate Editor

Accepted March 14, 2008 